\title{
Neonatal Culture Positive Sepsis, AE
}

National Cancer Institute

\section{Source}

National Cancer Institute. Neonatal Culture Positive Sepsis, AE. NCI Thesaurus. Code

C154927.

An adverse event in a newborn characterized by a systemic inflammatory response to an infection with an identifiable cause. 